\title{
SEARCH FOR FAINT GALAXIES TOWARDS NEARBY VOIDS
}

\author{
U. HOPP, B. KUHN, H. ELSÄSSER, K. BIRKLE and U. THIELE \\ MPI für Astronomie \\ D-69117 Heidelberg \\ Germany
}

\section{Introduction}

Recent redshift surveys of the Nilson-, Zwicky-, and ESO-Uppsala galaxy catalogues revealed that the bright galaxies in these catalogues follow a spatial distribution where large empty regions are surrounded by sheet-like structures of galaxies and clusters (e.g. Giovanelli \& Haynes 1991). From an observer's point of view, one may simply ask if the voids in the above-mentioned galaxy maps are real or if they reflect special observational selection effects, e.g. in surface brightness, integral magnitude or diameter. These catalogues contain many bright galaxies $\left(M_{B} \sim M_{B}^{*}\right)$, but only very few faint objects $\left(M_{B} \geq M_{B}^{*}+3^{m}\right)$. Older work on the distribution of intrinsically faint galaxies, like irregular dwarfs, claimed that the dwarfs follow the distribution which is outlined by the giant galaxies.

For example, Thuan et al. (1987) studied the distribution of the Nilson dwarf galaxies which belong to the first CfA slice and concluded that the dwarfs are distributed like the giants. But really faint galaxies, with $M_{B} \geq-16^{m}$, were mapped only to velocities of $2000 \mathrm{~km} / \mathrm{s}$. Such velocity distances are small compared to the distances and dimensions of the nearest voids.

Some theories of galaxy formation (e.g. Deckel \& Silk 1985; White et al. 1987) request a more homogeneous distribution of the mass than is outlined by the bright galaxies. They especially predict that dwarf galaxies fill the voids and show smaller spatial variations. Further, some theories predict that low surface brightness disk giants should form in voids (e.g. Hoffman et al. 1992).

\section{Our Approach}

Our idea was to search for such hitherto undetected galaxies in nearby voids. We define as nearby a velocity of less than $7500 \mathrm{~km} / \mathrm{s}$ for the centre of the void, but we finally concentrated on three voids with velocities around $3500 \mathrm{~km} / \mathrm{s}$. These voids were selected from published cone diagrams of bright galaxies. A fourth field was observed towards a well-known cluster of galaxies (A2151) to control our selection criteria in a well-known field. The field towards A2151 includes an additional nearby void. In these four fields we tried to search non-giant type galaxies like dEs, Irregulars, and low surface brightness galaxies. 
If the only observational bias is the limiting surface magnitude, then one should search on the new, deep Schmidt survey plates as done by others (see e.g. Eder et al. 1989; Schombert et al. 1992; or Phillipps in these proceedings).

If the observational bias is the diameter or a combination of diameter and surface brightness, one should improve the scale. At least the scale length of irregular dwarfs decreases with fainter absolute magnitude and the average central surface magnitude tends to be less luminous for fainter dwarfs (see e.g. Binggeli [1993] for cluster members and Hopp \& Schulte-Ladbeck [1993] for isolated objects). Therefore, one has to account for very small diameters and low surface brightness.

We therefore decided to use the Prime focus of our $3.5 \mathrm{~m}$ Calar Alto telescope to supplement the Schmidt surveys for possible void galaxies. We intended to profit from the better resolution for morphological classification of small galaxies, similar to the studies of the Virgo and Fornax clusters. For comparison, we also searched for faint galaxies on Schmidt plates in a larger field surrounding the Prime focus fields, up to now still on the old POSS.

Deep, one square degree photographic plates were obtained towards the centre of two voids in blue $\left(B_{\text {lim }} \sim 23^{m}\right)$ and red $\left(R_{\text {lim }} \sim 22^{m}\right)$ on IIIa emulsions. The third void was observed with a large Tektronix CCD to even fainter magnitudes, covering a field of about one square degree. The search on the POSS plates was done in fields of about 70 square degrees. The Prime focus plates were searched by eye for galaxies with the following selection criteria: the galaxies should always be larger than 5 arcsec and of late-type or dE-like morphology or with LSB features. If one would apply no other selection criterion, like diameter or magnitude, the resulting catalogue will be largely dominated by background galaxies with magnitudes around $M_{B}^{*}$ while it will contain only very few nearby dwarfs (e.g. Dickey 1988).

Galaxies with diameters between 5 and 3 arcsec are difficult to classify by eye. Therefore, we digitized our blue plates with a PDS microdensitometer and will search and classify with objective criteria for galaxies in this range. Further, we shall determine our selection effects and completeness with these objective algorithm.

The search criteria for the POSS fields were similar, but the diameter was restricted to 0.2 arcmin. By eye, a total number of $20,18,26$ and 25 high priority galaxies were selected in the prime focus images and 102, 73 and 109 galaxies on the POSS plates.

The follow-up spectroscopy was obtained with the Twin and Focal-reducer spectrographs of the Calar Alto $3.5 \mathrm{~m}$ telescope for the Prime focus sample and with the ESO/MPG $2.2 \mathrm{~m}$ telescope and EFOCS2 for the POSS sample. Low resolution spectra were obtained with a wavelength coverage between $370 \mathrm{~nm}$ and $950 \mathrm{~nm}(3.5 \mathrm{~m})$ or $350 \mathrm{~nm}$ and $600 \mathrm{~nm}(2.2 \mathrm{~m})$. Redshifts were obtained for 138 objects with a typical precision of $\pm 100 \mathrm{~km} / \mathrm{s}(3.5 \mathrm{~m})$ or $\pm 200 \mathrm{~km} / \mathrm{s}(2.2 \mathrm{~m})$. For all selected objects, CCD photometry was obtained in B and R. The observed galaxies have magnitudes in the range $-21 \leq M_{B} \leq-15$, indicating that our sample still contains some background giants. The central surface magnitudes are $18.5 \leq \mu[\mathrm{mag} / \mathrm{sqrarcsec}] \leq 25.5$, the majority between 21.5 and 24.0. A galaxy with an absolute magnitude of $-16^{m}$ can be mapped to a velocity distance of $7000 \mathrm{~km} / \mathrm{s}$ (Prime) and $5000 \mathrm{~km} / \mathrm{s}$ (POSS), respectively. About $2 / 3$ of our sample galaxies have emission lines. 


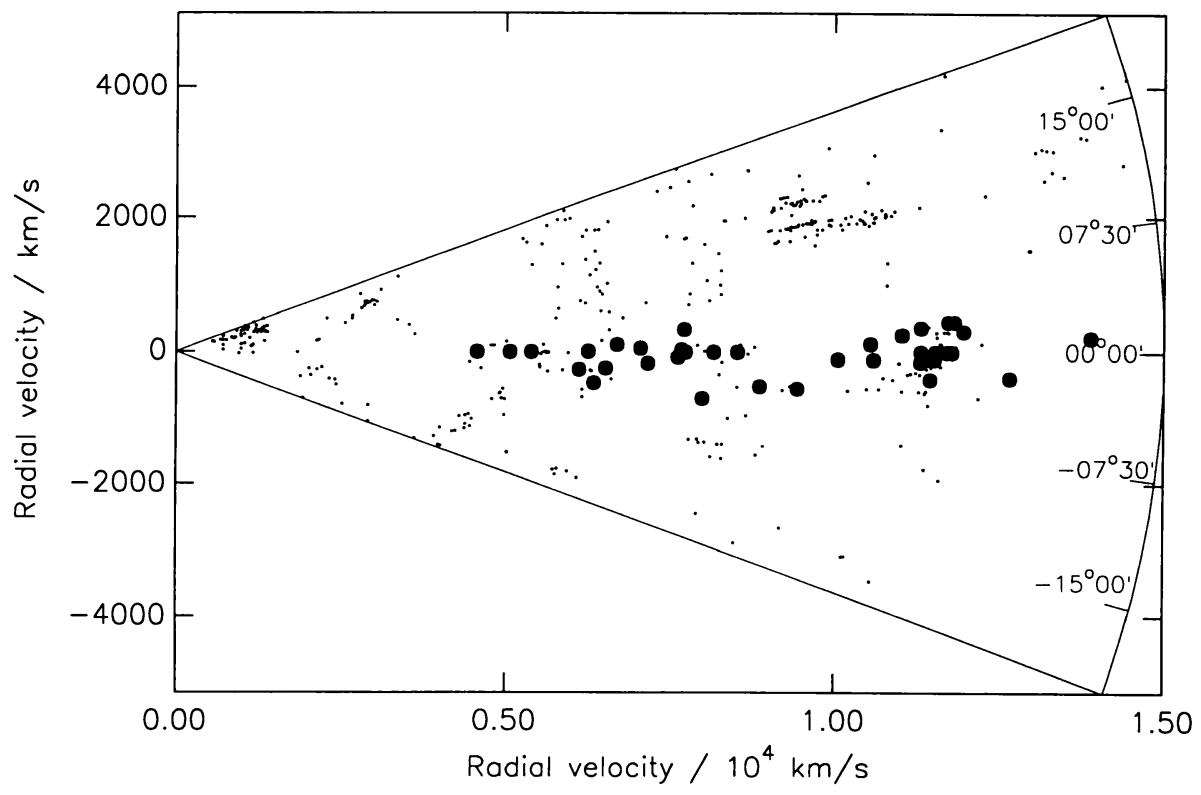

Figure 1. Cone diagram of one of our void-fields which contains three individual voids (see text). The small dots show the distribution of 361 CfA galaxies which outline the distribution of the normal giants. The CfA values are taken from the interval R.A. $=10^{n} 39^{m} \pm 5^{\circ}$, the range in declination is indicated to the right. The large dots mark the positions of the galaxies identified in our survey. In the central regions of the three visible voids, no homogeneously distributed galaxy population shows up. In the void centred at $7200 \mathrm{~km} / \mathrm{s}$, the outskirts are populated by star forming dwarfs which are newly identified in our program.

\section{Results}

The results for one of our three void-fields is outlined in Fig. 1, the results for the other fields are similar. Each field shows several individual voids, the example shown in Fig. 1 contains voids at the central velocities of 3100,7200 and $9800 \mathrm{~km} / \mathrm{s}$ respectively. We compare the distribution of the CfA galaxies (e.g. Huchra et al. 1990) with our own samples. There are obviously no galaxies which form a homogeneously population in the nearest void - even we should have seen them if there are some with magnitudes brighter than about $M_{B} \sim-15^{m}$. In the second void in the CfA distribution (at $7200 \mathrm{~km} / \mathrm{s}$ ), some of our galaxies populate the outskirts of the void at the far and nearby end. The central part of the void remains free of galaxies. About $80 \%$ of the galaxies which surround this second void are star forming dwarfs. We did not find any dEs or LSB giants. Similar results were found for other voids by Salzer (1989) who studied the distribution of emission-line galaxies. Our results are in good agreement with Schmidt-telescope based searches for late type or LSB-type galaxies (Eder et al. 1989; Schombert et al. 1992). These surveys were restricted to diameters of 24 arcsec and 30 arcsec, respectively. With our improved diameter criterion, we found a similar population of galaxies at similar distances as Eder et al. One should mention that up to now, the faintest end of the galaxy luminosity function is not mapped by any survey. 


\section{References}

Binggeli, B., 1993. Basel Preprint 64, 'Panchromatic View of Galaxies - Their Evolutionary Puzzle', Kiel, 1993.

Deckel, A. and Silk, J., 1986. Astrophys. J., 303, 39.

Dickey, J.M., 1988. Astron. Soc. Pac. Conf. Ser., 5, 9.

Eder, J.A., Schombert, J.M., Deckel, A. and Oemler, A., 1989. Astrophys. J., 340, 29.

Giovanelli and Haynes, 1991. Ann. Rev. Astron. Astrophys., 29, 499.

Hoffman, Y, Silk, J. and Wyse, R.F.G., 1992. Astrophys. J., 388, L13.

Hopp, U. and Schulte-Ladbeck, R.E., 1993. In preparation.

Huchra, J.P., Geller, M.J., de Lapparent, V. and Corwin, H.G., 1990. Astrophys. J., 72, 433.

Salzer, J.J., 1989. Astrophys. J., 347, 152.

Schombert, J.M., Bothun, G.D., Schneider, S.E. and McGaugh, S.S., 1992. Astron. J., 103, 1107.

Thuan, T.X., Gott, J.R. and Schneider, S.E., 1987. Astrophys. J., 315, L93.

White. S.D.M., Davis, M., Efstathiou, G. and Frenk, C.S., 1987. Nature, 330, 451. 